\title{
AN ADAPTIVE RADIO ACCESS NETWORK RESOURCE MANAGEMENT TECHNIQUE FOR A HSDPA NETWORK
}

\author{
Xinzhi Yan \\ University of Newcastle \\ Newcastle, Australia
}

\author{
Jamil Y. Khan \\ University of Newcastle \\ Newcastle, Australia
}

\author{
Brendan Jones \\ SingTel OPTUS \\ Sydney, Australia
}

\section{ABSTRACT}

This paper presents an adaptive bandwidth management technique to improve the transmission efficiency of a High Speed Downlink Packet Access (HSDPA) network by adaptively allocating radio access network resources. The radio access network resources are allocated based on the air interface demand. The proposed bandwidth management technique is evaluated by an analytical and a simulation model. Both analytical and simulation results show that the proposed algorithm improves the $\mathrm{I}_{\mathrm{ub}}$ link utilization, and keep high the HSDPA air interface resource utilization by jointly optimizing the resource allocation processes.

\section{INTRODUCTION}

As one of the initial steps of the 3GPP Long Term Evolution (LTE) plane, the HSDPA standard has been introduced to significantly improve the downlink capacity. The HSDPA architecture was initially introduced in the $3 \mathrm{GPP}$ release 5. The HSDPA channel uses a short $2 \mathrm{~ms}$ transmission time interval (TTI), and uses variable coding and modulation techniques to achieve high data rate. In addition to above techniques the Node-B based fast scheduling and fast physical layer hybrid automatic repeat request (H-ARQ) techniques are used in the HSDPA standard. The HSDPA architecture uses a downlink buffer in the Node-B. The buffer along with a scheduler that shares the transmission resources enables a higher peak transmission rate for HSDPA users on a radio link. A flow control is generally employed on the $I_{u b}$ link between a RNC (Radio Network Controller) and a Node-B to avoid buffer overflow or buffer starvation. According to the HSDPA roadmap within next few years the air interface data rates could reach up to $14.4 \mathrm{Mbps}$ and eventually the data rate could reach $100 \mathrm{Mbps}$ under the LTE plan. In a HSDPA network user connection rate could vary because of transmission conditions and also due to time and code sharing among users. The peak data rate requires extremely favorable radio conditions and it is unlikely that all users in a cell would be able to get such a high data rate. Within limits to improve effective data rate on the air interface it is necessary to match radio access network (RAN) parameters with the air interface characteristics. For high data rate wireless networks it is necessary to manage radio and RAN resources in order to optimize the overall HSDPA network efficiency. Since the air interface throughput varies with the transmission channel condition it will be necessary to adaptively allocate the $I_{u b}$ link bandwidth according to the air interface demand. In a HSDPA network if an $\mathrm{I}_{\mathrm{ub}}$ link cannot provide high enough instantaneous bandwidth then sufficient number of packets cannot be supplied to the Node-B from the RNC hence, it may be possible that there will not be enough packets in the Node-B buffer to sustain a peak transmission rate on the air interface [1]. On the other hand if a higher capacity is allocated on the $\mathrm{I}_{\mathrm{ub}}$ link then the RAN resources will remain under utilized. Typically, in most of research works the $\mathrm{I}_{\mathrm{ub}}$ link resource allocation issue is not considered by the HSDPA flow control. High HSDPA traffic intensity could increase the risk of congestions on a RAN leading to increased packets delays and higher probability of packet loss, which in turn could trigger RLC (Radio Link Control) layer retransmissions subsequently degrading the QoS of a HSDPA connection [2]. The 3GPP document contained in the reference [3] presented a HSDPA congestion control technique to improve the traffic flow on the $I_{u b}$ link. But the suggested congestion control technique cannot act as a flow control to pro-actively prevent an imminent congestion rather used as an "emergency break" in order to keep the system at a stable state.

Several recent research works tried to address the issue. Bajzik et. al. [2] introduced a cross-layer backpressure algorithm to prevent congestions and the RLC layer retransmissions. Legg [4] presented an $\mathrm{I}_{\mathrm{ub}}$ link flow control strategy; the work found that the scheduler in the Node-B has a major influence on Node-B memory usage. Necker et. al. [5] investigated the impact of the $I_{u b}$ flow control on the HSDPA system performance, the work indicated that a larger protocol delay and the resource grant update delay between the Node-B and the RNC could deteriorate the IP packet delay characteristic. In this paper we propose an adaptive RAN resource technique to improve the HSDPA air interface and $I_{u b}$ link utilizations. The algorithm adaptively allocate RAN resources by estimating the HSDPA air interface throughput to pro-actively prevent any congestion, and to optimize the both air interface and RAN performances to improve the overall QoS of HSDPA connections.

The paper is organized as follows. Section II studies the interdependence between HSDPA air interface performance and the $\mathrm{I}_{\mathrm{ub}}$ capacity using an analytical model. The analytical model examines the interaction between the air interface and RAN parameters to jointly optimize the HSDPA network performance. Section III describes the proposed adaptive RAN resources allocation technique. The simulation model is introduced in the section IV. Simulation results are presented in section V. Conclusions are drawn in the section VI.

\section{INTERDEPENDENCE BETWEEN I IB BANDWIDTH AND HSDPA PERFORMANCE}

In this section, we present an analytical model using the Markov chain model to investigate the interdependence between the $I_{u b}$ link capacity and the air interface performance. It is necessary to employ a flow control algorithm between the Node-B and the RNC to reduce the possibilities of a buffer overflow and poor link utilization. Fig. 1 shows the basic operation of the proposed flow control 
algorithm operating between a Node-B and a RNC. As shown in the figure that the RNC maintains a large buffer of packets which is updated via the core network. The Node-B maintains a minimum buffer which will enable peak transmission rate for HSDPA users on the air interface. The flow control algorithm continuously updates the Node-B buffer based on the HSDPA connection throughput and the connection demand.

For each HSDPA user we allocate a fixed space in the Node-B buffer. We assume that $n$ represents the buffer size for each user in the Node-B buffer, where $n$ is represented in number of MAC-d PDUs. The value of $n$ is equal to total number of MAC-d PDUs that could be sent over the air interface at the peak rate in every TTI $(2 \mathrm{~ms})$ under the best transmission channel condition. We assume that the $\mathrm{I}_{\mathrm{ub}}$ link bandwidth is $m$, where $m$ is the number of MAC-d PDUs sent in each TTI over the link. After every transmission opportunities on the air interface, the Node-B immediately requests the RNC to send more MAC-d PDUs to replenish its buffer. The request size $R_{s}$ in number of PDUs is equal to the free buffer size. If the request size is larger than the $\mathrm{I}_{\mathrm{ub}}$ bandwidth $m$ i.e. $R_{s}>m$, then to avoid the $\mathrm{I}_{\mathrm{ub}}$ link congestion which could lead to a long buffer update delay and HSDPA packet drop, only $m$ MAC-PDUs are delivered to the Node-B. The value of $R_{s}$ could vary between 1 to $n$. When $m \geq n$, there are not impact of $\mathrm{I}_{\mathrm{ub}}$ link constraint on HSDPA performance. In the following, we make analysis under $m<n$. Using the above flow control algorithm the Node-B buffer is used to adjust the HSDPA user throughput on the air interface based on the radio link condition. It is possible that a peak air interface transmission rate may not be sustainable due to $I_{u b}$ link and the air interface transmission rate mismatch.

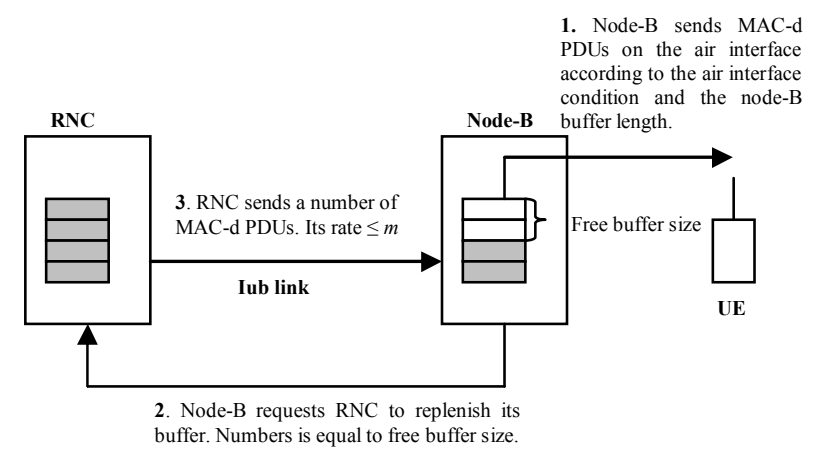

Figure 1. the flow control between Node-B and RNC

Due to time-variant nature of radio channels, the transmission data rate of the air interface is highly variable. We assume that the transmission rate (expressed in number of MAC-d PDUs of per TTI) of a HSDPA connection is distributed between 0 and to a maximum number $n$. The value of $n$ depends on the UE category. Let $r$ represent the air interface rate, which the radio channel condition can support in terms of PDU. Let $R_{i}$ represents the probability of air interface transmission rate, where $i$ represent the number of PDUs transmitted at that rate. After the Node-B transmit PDUs on the air interface, it is assumed that the Node-B buffer immediately request RNC to send packets to replenish its buffer before the next transmission opportunity. Let $l_{n}$ be
MAC-d PDU numbers in Node-B buffer measured after the buffer replenished and waiting for the next transmission opportunity over the air interface in $n$-th TTI. The buffer length could vary between $m$ and $n$, i.e. $m \leq l_{n} \leq n$. We can form a Markov chain using the values of $l_{n}$. Consider the stationary state where all $\left\{l_{n}\right\}$ have the same probability distribution. Let $l$ denote the random variable in MAC-d PDU numbers in Node-B buffer.

$$
\text { Let, } \pi_{i}=P\{l=m+i\}, \quad i=0, \ldots, n-m
$$

The transition matrix $P$ is shown in (2).

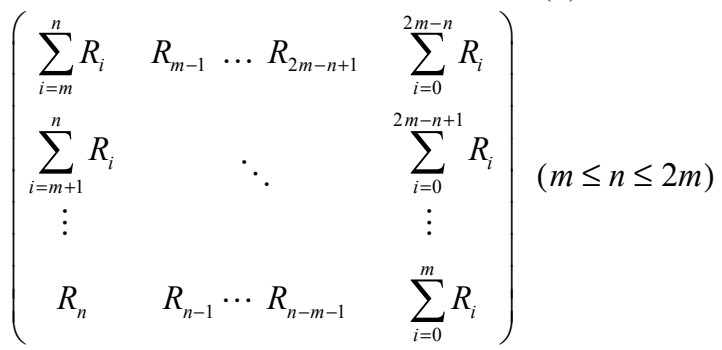

In the stationary state, we can write $\pi=\pi P$, so

$$
\begin{aligned}
& \pi_{0}=\pi_{0} \sum_{i=m}^{n} R_{i}+\pi_{1} \sum_{i=m+1}^{n} R_{i}+\ldots .+\pi_{n-m} R_{n} \\
& \pi_{1}=\pi_{0} R_{m-1}+\pi_{1} R_{m}+\ldots .+\pi_{n-m} R_{n-1} \\
& \vdots \\
& \pi_{n-m-1}=\pi_{0} R_{2 m-n+1}+\pi_{1} R_{2 m-n}+\ldots .+\pi_{n-m} R_{n-m-1} \\
& \pi_{n-m}=\pi_{0} \sum_{i=0}^{2 m-n} R_{i}+\pi_{1} \sum_{i=0}^{2 m-n+1} R_{i}+\ldots .+\pi_{n-m} \sum_{i=0}^{m} R_{i} \\
& \pi_{0}+\pi_{1}+\cdots+\pi_{n-m}=1
\end{aligned}
$$

We present a brief analysis to calculate the HSDPA air interface resource utilization. We define the HSDPA air interface resource utilization $\eta$ as the ratio of actual number of MAC PDUs (which is limited by number of PDUs in Node-B buffer) that can be transmitted via the air interface to the maximum number of MAC PDUs that can be supported by air interface condition per TTI, as shown in (4). The HSDPA air interface utilization takes account of the radio channel condition, buffer length and the $I_{u b}$ link effective bandwidth. For example, on one occasion, if 8 PDUs of a HSDPA connection is in Node-B buffer but the radio link condition permits transmission of 10 PDUs then we obtain a $\eta$ value of $0.8(8 / 10)$. In that case the when transmission opportunity arises then the Node-B can not transmit sufficient number PDUs.

$$
\eta=\frac{\text { Actual_MAC_PDU_Tx }}{M a C_{-} M A C_{-} P D U_{-} \text {air }}
$$

We calculate HSDPA air interface utilization $\eta$ with (4). When $l=m$,

$$
\begin{aligned}
& \text { if } r \leq m, \eta_{l=m}=100 \% \text {; } \\
& \text { if } r=m+1, \eta_{l=m}=\frac{m}{m+1} \text {; } \\
& \vdots \\
& \text { if } r=n, \eta_{l=m}=\frac{m}{n} ;
\end{aligned}
$$




$$
\begin{aligned}
& \text { When } l=n-1 \text {, } \\
& \text { if } r \leq n-1, \eta_{l=n-1}=100 \% \text {; } \\
& \text { if } r=n, \eta_{l=n-1}=\frac{n-1}{n} \text {; } \\
& \text { When } l=n \text {, } \\
& \eta_{l=n}=100 \% \text {; }
\end{aligned}
$$
by:

So, the average HSDPA air interface utilization $\eta$ is given

$$
\begin{aligned}
\eta= & \pi_{0}\left(\sum_{i=0}^{m} R_{i} \times 1+R_{m+1} \times \frac{m}{m+1}+\ldots .+R_{n} \times \frac{m}{n}\right)+ \\
& \pi_{1}\left(\sum_{i=0}^{m+1} R_{i} \times 1+R_{m+2} \times \frac{m+1}{m+2}+\ldots .+R_{n} \times \frac{m+1}{n}\right) \\
& +\ldots .+\pi_{n-m} \times 1
\end{aligned}
$$

From the statistical point of view, the air interface capacity of a cell will be approximately normally distributed according to the Central Limit Theorem. Since the distribution of air interface rate is discrete, to simplify our analysis, we approximate the HSDPA air interface data rate using the following binomial distribution (6).

$$
\begin{aligned}
& R_{i}=\left(\frac{n}{i}\right) p^{i}(1-p)^{n-i} \quad 0<i \leq n ; \quad 0<p \leq 1 \\
& \text { mean }=n \cdot p \\
& \text { HSDPA average throughput at RLC layer } \\
& =n \times p \times R L C \text { Packet_size/TTI }
\end{aligned}
$$

Where $R_{i}$ represents the probability of transmission rate air interface support, where $i$ represent the number of PDUs transmitted at that rate and $n$ is equal to the maximum number of MAC-d PDUs that can be sent in every TTI. $n$ is up to UE category. The value of $p$ depends on cell parameters. We assume that a very good and controlled coverage in a small cell and low user mobility could lead to higher value of $p$, which represents higher air interface transmission rate for users. Best radio channel condition is represented by the value of $p=1$.

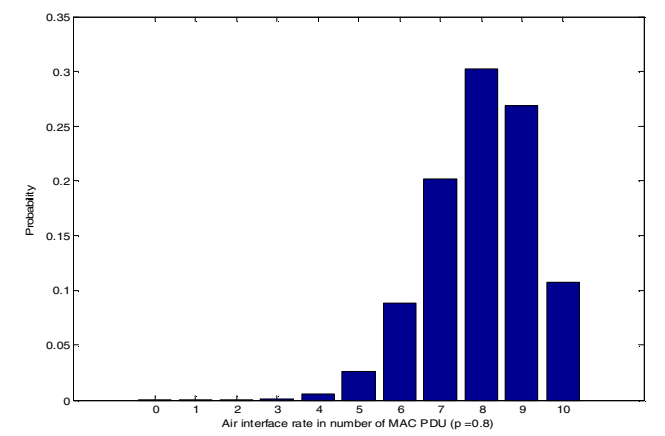

Figure 2. Probability distribution of air interface represented in number of MAC-d PDUs for $p=0.8$ and $\mathrm{n}=10$.

Fig. 2 shows the probability distribution of air interface transmission rate for $p=0.8$, and $n=10$ for a category $12 \mathrm{UE}$. This plot shows that for $p=0.8$ a HSDPA link may transmit a number of PDUs per TTI at different times. The plot shows that for $p=0.8$ there are high probabilities that a UE will transmit 7 to 9 PDUs per TTI. The peak of the plot will shift towards left for lower values of $p$. Similar results were reported by an Ericsson research group who measured HSDPA network performances in different transmission conditions [6].

Using the above analytical technique, we can calculate the HSDPA transmission utilization for a category $12 \mathrm{UE}$ with different air interface conditions and $\mathrm{I}_{\mathrm{ub}}$ link capacity. Analysis results are shown in Fig.3. The figure shows that the value of $\eta$ increases with the $\mathrm{I}_{\mathrm{ub}}$ data rate. The figure also shows that the degree of improvement depends on the air interface condition. In a realistic HSDPA network the air interface throughput will vary depending on the transmission channel condition. Hence, a fixed $\mathrm{I}_{\mathrm{ub}}$ bandwidth allocation will either reduce the air interface utilization or it will reduce the $\mathrm{I}_{\mathrm{ub}}$ link utilization. To avoid above problems we propose a simple but effective RAN resource allocation technique to avoid above problems. Fig. 3 shows that for an HSDPA connection the HSDPA air interface utilization could reach $97 \%$ when the $I_{u b}$ link effective bandwidth is increased to its air interface average throughput. In the other word, if a HSDPA connection's air interface average throughput is known, and the $\mathrm{I}_{\mathrm{ub}}$ link bandwidth is allocated accordingly then the air interface utilization could reach as high as $97 \%$.

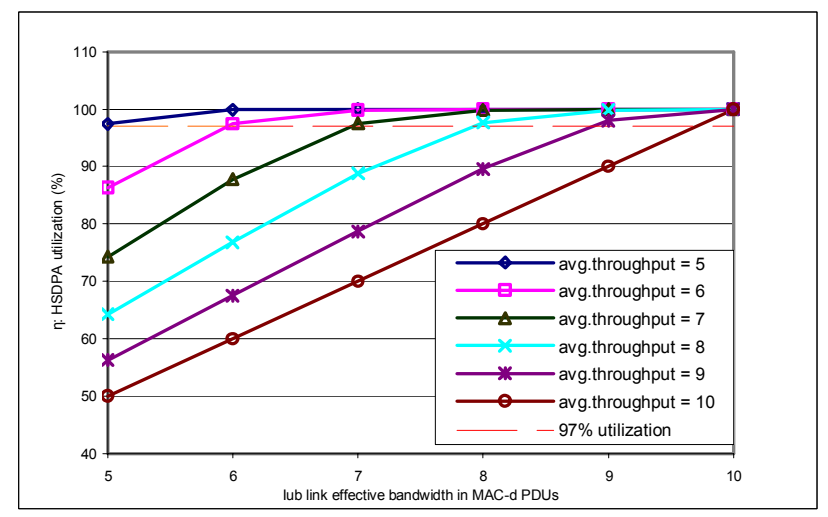

Figure 3. the interdependence between Iub effictive bandwidth and HSDPA air interface utilization under different HSDPA average throughput supported by air interface (in MAC-d PDUs per TTI)

\section{Adaptive Management Algorithm}

Based on above observations, we propose a simple adaptive $\mathrm{I}_{\mathrm{ub}}$ link bandwidth allocation technique based on the air interface average throughput. The adaptive resource management algorithm will be located at Node-B which will measure every HSDPA connection throughput and using the measured information it will allocate the corresponding connection data rate on the $\mathrm{I}_{\mathrm{ub}}$ link.

\section{A. Estimating HSDPA air interface average throughput}

We estimate each HSDPA connection average throughput using a simple averaging equation. Equation (9) shows the averaging process. The connection throughput is measured for every bursts and the running average of the air interface throughput is used to allocate the $\mathrm{I}_{\mathrm{ub}}$ link bandwidth for that particular connection. The average throughput value is calculated for every data bursts separately i.e. when 
connection goes to the idle mode then its average throughput value is reset to the default value of $m$.

$$
\overline{\text { throughput }}=\frac{1}{N} \sum_{i=1}^{N} X_{i}
$$

Where $X_{i}$ represents the throughput in number of MAC PDUs for the $i$-th transport opportunity of a connection and $n$ represents the transport opportunity numbers.

when $X_{1}, X_{2}, \ldots . X_{i} \sim \operatorname{binomial}(n, p)$ as (6),

Round Robin scheduling technique is used,

$$
\begin{aligned}
& \operatorname{var}(\overline{\text { throughput }})=\operatorname{var}\left(\frac{1}{N} \sum_{i=1}^{N} X_{i}\right) \\
& =\left(\frac{1}{N}\right)^{2} \sum_{i=1}^{N} \operatorname{var}\left(X_{i}\right)=\left(\frac{1}{N}\right)^{2} N n p(1-p)=\frac{n p(1-p)}{N}
\end{aligned}
$$

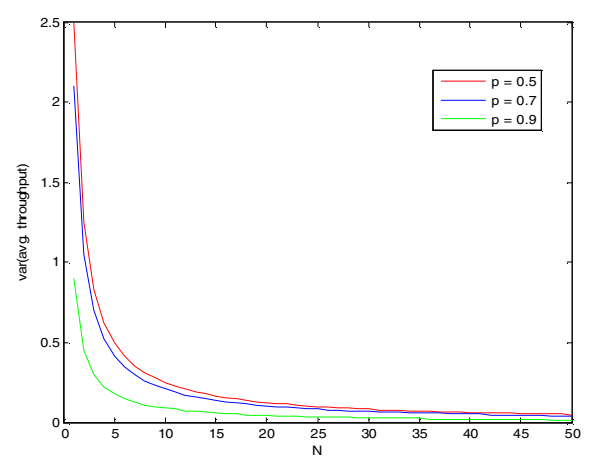

Figure 4. the variance of average throughput $(n=10)$

As shown in Fig. 4, with the increasing value of $N$ the calculated value of a connection throughput converges. Fig. 5 shows the estimation process. As shown in the figure that the HSDPA connection throughput varies quite considerably over the $100 \mathrm{~ms}(50 \mathrm{TTI})$ which is a snapshot of a connection throughput. If the RNC to Node-B connection bandwidth is allocated based on the instantaneous throughput of the air interface then the air interface utilization as well as the $\mathrm{I}_{\mathrm{ub}}$ link utilization could drop significantly. Whereas if we use the running average value of the HSDPA throughput then the both links throughput will be matched thus increasing the overall utilization.

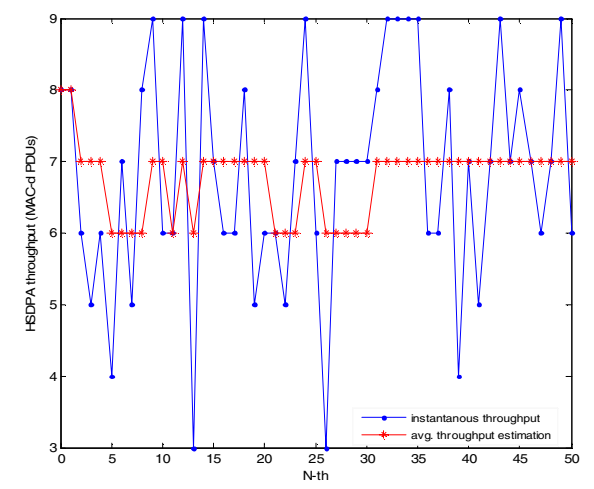

Figure 5. Instantaneous air interface throughput and the running average througput value.

\section{B. Adaptive Iub bandwidth allocation}

The scheduler in Node-B selects an active HSDPA connection to transmit packet on the air interface in every TTI. After each transmission the Node-B calculates the $\mathrm{I}_{\mathrm{ub}}$ connection bandwidth $\bar{m}$ using (9). The Node-B also calculates the value of $R_{s}$ by measuring the buffer length. Node-B then sends a control message by incorporating the value of the $\mathrm{I}_{\mathrm{ub}}$ connection bandwidth and the request size $R_{s}$. Using the above $\mathrm{I}_{\mathrm{ub}}$ bandwidth allocation technique it is possible to match the air interface throughput rate and the $\mathrm{I}_{\mathrm{ub}}$ link throughput. The required aggregate $\mathrm{I}_{\mathrm{ub}}$ link bandwidth is calculated by (11).

$$
\text { Bandwidth }=\sum_{i=1}^{N_{\text {total }}} s_{i} \times \overline{m_{i}}
$$

Where $s_{i}$ represents the HSDPA connection $i$ repetition rate that depend on the scheduling technique used by the Node-B. $N_{\text {total }}$ represents the total number of HSDPA connections mapped on the $\mathrm{I}_{\mathrm{ub}}$ link

\section{Simulation Model}

A simulation model has been developed to investigate the dependence of the HSDPA air interface throughput and the $I_{u b}$ link characteristics. The simulator is consists of an $\mathrm{I}_{\mathrm{ub}}$ interface and a physical link, a traffic generator, an air interface link and the proposed flow controller as shown in the Fig. 6 [7], [8]. We assume that the Core Network (CN) can deliver enough packets to the RNC so that sufficient number of packets waiting in the RNC buffer during the active period of a data burst. The flow controller is implemented according to the description of the section II and III. The Node-B allocates each HSDPA connection a transmission bandwidth according to the radio channel condition which is simulated by the binomial distribution as shown in (6). The $\mathrm{I}_{\mathrm{ub}}$ link is simulated using the AAL2/ATM and UDP/IP transport protocols.

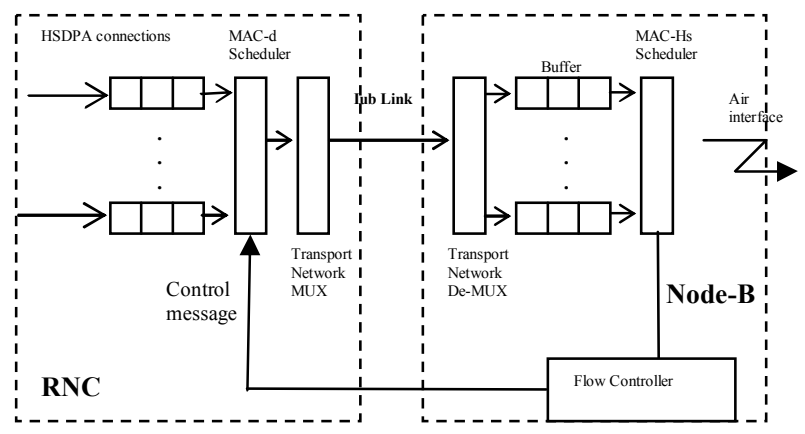

Figure 6. Simulation moddel architecture.

\section{Simulation Results}

Using the above simulation model we obtain several results. First we compare simulation results with analytical results from section II. In the simulation, we use an E1 physical link between RNC and Node-B. Transport layer protocol is AAL2/ATM. The maximum buffer size for a category $12 \mathrm{UE}$ is 10 MAC-d PDUs. Fig. 7 shows the HSDPA air interface 
utilization $\eta$ for different average air interface throughput. The air interface downlink throughput is represented in terms of number of MAC-d PDUs transmitted per TTI. The figure shows both the analytical and simulation results. Comparing the analytical and simulation results we find that both models are in close agreement. With the increasing downlink throughput under good channel conditions the value of $\eta$ decreases because of the buffer starvation problem.

Next we compare the HSDPA air interface utilization figures for different $I_{u b}$ link bandwidth allocation technique. In this simulation we used total 15 categories 12 UEs. We grouped these 15 UEs in to three groups with each group of 5 UEs. One group assumed to work in good channel conditions which support an average of 9 MAC-PDUs per TTI. The second group of 5 UEs is assumed to operate in medium channel conditions with an average of 7 MAC-PDUs per TTI. The third group consists of 5 UEs operating in poor channel conditions with an average throughput of 5 MAC-PDUs per TTI. In this simulation we used a Round Robin (RR) scheduling technique at the Node-B. For this simulation first we allocate effective $I_{u b}$ link bandwidth as air interface peak rate. For category $12 \mathrm{UE}$, its peak rate is $10 \mathrm{MAC}-\mathrm{d}$ PDU per TTI. In the next simulation run we use the adaptive $I_{u b}$ link bandwidth allocation as described in section III. Fig. 8 shows the HSDPA air interface utilization among UEs group. Result shows that the adaptive algorithm only reduces HSDPA air interface utilization by about $3 \%$. This value is similar to analytical value in section II. Next we compare the $I_{u b}$ physical link bandwidth requirements and the effective bandwidth utilization for the adaptive allocation and peak rate allocation techniques using the AAL2/ATM and UDP/IP transport protocols. Fig. 9 shows the result. Figure shows that to support same amount of air interface traffic the adaptive allocation technique requires about $0.8 \mathrm{Mbps}$ less $\mathrm{I}_{\mathrm{ub}}$ link data rate for the AAL2/ATM transport protocol and about 0.7 Mbps less data rate for the UDP/IP protocol. Also, if we compare the link utilization figures then we find that the adaptive algorithm increases the link utilization by $27 \%$. We obtain this improvement using the simple adaptive algorithm to match the air interface throughput with the $\mathrm{I}_{\mathrm{ub}}$ link data rate.

\section{CONCLUSION}

In this paper initially we investigated the interdependence between HSDPA radio resource utilization and $I_{u b}$ link bandwidth. By studying the interdependency between downlink and the $I_{u b}$ link characteristics we developed a simple adaptive $I_{u b}$ link bandwidth allocation technique. We presented both analytical and simulation models to study the performance of both interfaces. Above results show the adaptive algorithm clearly reduces the radio access network resource requirements while keeping the air interface throughput high. Simulation and analytical results clearly shows that for high data rate HSDPA connections it is necessary to jointly optimise the air interface and radio access network radio resources.

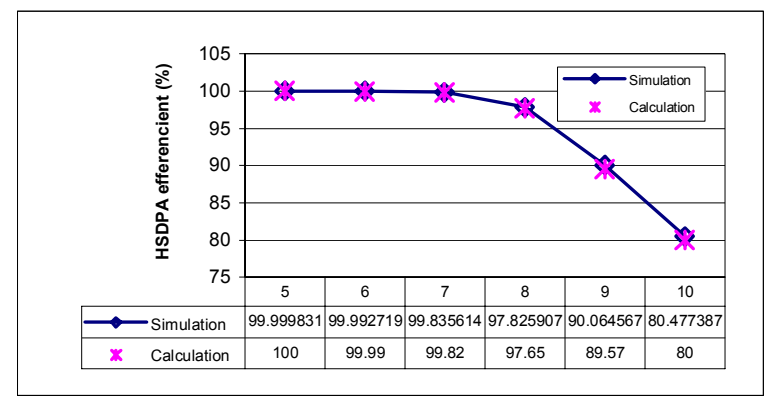

Figure 7. HSDPA utilization for different average throughput (in MAC-d PDUs per TTI) supported by air interface (calculation vs. simulation)

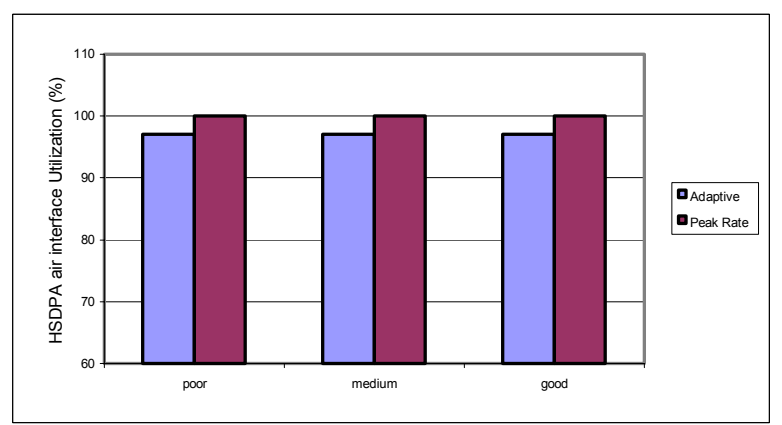

Figure 8. HSDPA radio resource utilization (adpative allocation vs. peak rate allocation).

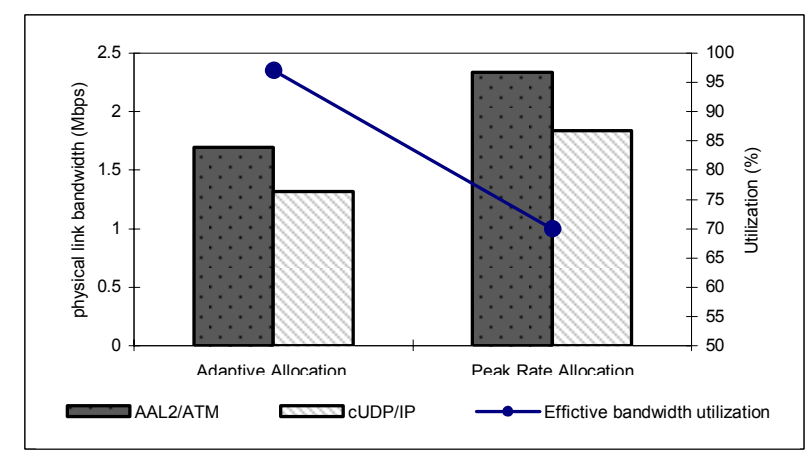

Figure 9. Iub physical link bandwidth requirment and effictive bandwidth utilization

\section{REFERENCES}

[1] H. Holma, A. Toskala, HSDPA/HSUPA for UMTS, John Wiley and Sons, 2006, pp.26-27

[2] L. Bajzik, L. Korossy, K. Veijalainen, and C. Vulkan, "Cross-Layer Backpressure to Improve HSDPA Performance," Proc. PIMRC 2006, helsinki, Finland, September 2006.

[3] 3GPP, TR25.902 v7.0.0, "Iub/Iur congestion control (Release 7),".

[4] P.J.Legg, "Optimised Iub flow control for UMTS HSDPA" in Proc. IEEE VTC 2005-Spring, Stockholm, Sweenden, Jun 2005.

[5] M. C. Necker and A. Weber, "Impact of lub flow control on HSDPA system performance," Proc. PIMRC 2005, Berlin, Germany, September 2005

[6] J.Derksen, et. al. "HSDPA performance and evolution", Ericsson Review, Vol.3, 2006.

[7] 3GPP TS 25.308 v6.3.0 (2004-12), "High Speed Downlink Packet Access (HSDPA); Overall description; Stage 2 (Release 6)".

[8] 3GPP TS 25.435 v6.3.0, "UTRAN Iub Interface User Plane Protocols for Common Transport Channel data streams (Release 6)". 